Thamnolaea albiscapulata (Rüpp.): Fischer, Ztschr. f. d. ges. Orn. 1884, p. 303. wascha.

468. Myrmecocichla aethiops (Licht.). Mossiro, Nai-

469. Myrmecocichla nigra (Vieill.) var. leucoluema Rchw. Nguru-Berge.

470. Monticola rufocinerea (Rüpp.). Naiwascha.

471. Monticola saxatilis (L). Bagamojo, Lamu, Maurui. wascha.

472. Saxicola isabellina Rüpp. Kipini, Barawa, Nai-

473. Saxicola oenanthe (L.). Klein-Aruscha.

474. Saxicola morio H. et Ehr. Lamu-Küste, Barawa.

475. Saxicola Schalowi Fschr. et Rchw. Naiwascha.

476. Campicola Livingstoni Trstr. Bagamojo, Pare, Aruscha, Mossiro, Ngaruka, Naiwascha.

Fschr. et Rchw., J. f. 0. 1879, p. 255: Campicola pileata Gm. 477. Pratincola rubicola (L.). Naiwascha.

478. Tarsiger orientalis Fchr. et Rchw. Pangani.

479. Lusciola africana Fschr. et Rchw. Klein-Aruscha.

\title{
Beiträge zur Ornis des nördlichen Illinois.
} Von

\section{- H. Nehrling.}

(Schluss; siehe Jahrgang 1883, S. 257.)

XXIX. FAM. TETRAONIDAE.

a. Genus Bonasa Steph.

153. B. umbellus Steph. Wald- oder Buschhuhn; Ruffled Grouse. Seltener Standvogel, aber nur im Walde.

b. Genus Cupidonia Reichenbach.

154. C. cupido Brd. Prairiehuhn; Pinnated Grouse, Prairie Chicken, Prairie Hen. Während meines Aufenthalts im nördlichen Illinois hatte ich oft Gelegenheit, das schöne Prairiehuhn genauer kennen zu lernen. Ich fand Nester bei Addison und Schaumburg (Du Page Co.) immer im hohen Grase der Prairie; das Gelege bestand meist aus 10 bis 12 Eiern, doch fand ich auch 15 Stück in einem Neste. Die Farbe der Eier ist hellbräunlich oder gelblich; oft sind sie mit ganz kleinen dunkelbraunen Pünktchen besäet. Von Jahr zu Jahr wird auch dieses interessante Huhn seltener. 
c. Genus Pedioccetes Baird.

155. P. phasianellus columbianus Coues. Spitzschwänziges Prairiehuhn; Sharp-tailed Grouse. Soll früher zahlreich gewesen sein, jetzt selten. Wird leicht mit dem vorigen verwechselt.

\section{FAM. PERDICIDAE. \\ Genus Orty $x$ Steph.}

156. O. virginianus Bonap. Baumwachtel; Quail, Partridge. Zahlreicher Standvogel.

XXXI. FAM. ARDEIDAE.

a. Genus Ardea Linné.

157. A. herodias L. Blauer Reiher; Great Blue Heron. In sumpfigen Waldgegenden ziemlich zahlreicher Brutvogel.

b. Genus Butorides Blyth.

158. B. virescens Bp. Grüner Reiher; Green Heron, Flyup-the Creek, Shytepoke. Der häufigste Reiher; brütet zahlreich.

c. Genus Botaurus Steph.

159. B. lentiginosus Steph. Rohrdommel; American Bittern, Stake Driver. Brutvogel in Sümpfen mit hohem Schilf, Binsen und Gebüsch bewachsen, zahlreich aber nur da, wohin selten der Fuss eines Menschen tritt.

\section{d. Genus Ardetta Gray.}

160. A. exilis Gray. Zwergrohrdommel; Least Bittern. Bewohnt besonders die Sümpfe (Sloughs) der Prairien.

XXXII. FAM. STREPSILIDAE.

a. Genus Strepsilas Illiger.

161. S. interpres Illig. Steinwälzer; Turnstone. Gewöhnlicher Vogel am Ufer des Michigansees.

XXXIII. FAM. CHARADRIIDAE.

a. Genus Charadrius Linné.

162. C. dominicus Müller. Goldregenpfeifer; Golden Plover. Während der Zugzeit nicht selten.

b. Genus Oxyechus Reichenbach.

163. O. vociferus Reich. Schreiregenpfeifer; Kill-deer. Zahlreicher Brutvogel in der fruchtbaren Prairie.

c. Genus Aegialitis Boie.

164. Aé. semipalmatus Bonap. Halbmondregenpfeifer; Semipalmated Plover. Nur auf dem Durchzug.

165. Ae. melodus circumcinctus Ridgw. Gürtel- oder Bandregenpfeifer; Belted Piping Plover. Brütet zahlreich. 


\section{FAM. SCOLOPACIDAE.}

a. Genus Philohela Gray.

166. P. minor Gray. Amerikanische Waldschnepfe; American Woodcock. Brutvogel, doch nirgends zahlreich.

b. Genus Gallinago Leach.

167. G. media Wilsonii Ridgw. Gewöhnliche Schnepfe; Common Snipe. Am zahlreichsten während der Zugzeit; in manchen Gegenden wahrscheinlich auch Brutvogel.

c. Genus Micropalama Baird.

168. M. himantopus Brd. Stelzer; Stilted Sandpiper. Nur während der Zugzeit und auch dann selten.

d. Genus Tringa Linné.

169. T. canutus Linn. Rothbrüstige Schnepfe; Robin Snipe. Während der Zugzeit.

\section{e. Genus Actodromas Kaup.}

170. A. maculata Coues. Gefleckter Strandläufer; Pectoral Sandpiper. Zahlreich in der Zugzeit.

171. A. minutilla Bp. Kleiner Strandläufer; Least Sandpiper. Auch von dieser während der Zugzeit zahlreichen Art dürften im nördlichen Illinois nur wenige Pärchen brüten.

f. Genus Ereunetes Illiger.

172. E. pusillus Cass. Halbmondstrandläufer; Semipalmated Sandpiper. Im Herbst beobachtet.

g. Genus Totanus Bechstein.

173. T. flavipes Vieill. Grün- oder Gelbschenkel; Lesser Yellowlegs. Zahlreicher Zugvogel; scheint gar nicht oder doch nur selten im nördlichen Illinois zu brüten.

h. Genus Rhyacophilus Kaup.

174. R. solitarius Cass. Einsiedlerstrandläufer; Solitary Sandpiper. Der zahlreichste Brutvogel der Familie, namentlich in Sümpfen der Wälder.

i. Genus Symphemia Rafinesque.

175. S. semipalmata Hartl. "Willet"; Willet. Ziemlich zahlreicher Brutvogel an passenden Oertlichkeiten.

k. Genus Bartramia Lesson.

176. B. longicauda Bp. Prairie- oder Bartramsregenpfeifer; Bartram's Tatler, Tield Plover, Upland Plover, Prairie Plover. Zahlreicher Brutvogel der ebenen Prairie in der Nähe des Wassers. 


\section{Genus Tringoides Bonaparte.}

177. T. macularius Gray. Gemeiner Strandläufer; Spotted Sandpiper. Brutvogel und zahlreich an grösseren Flüssen.

m. Genus Numenius Linné.

178. N. longirostris Wils. Brachvogel, Sichelschnäbler; Longbilled Curlew. Berührt gelegentlich des Durchzugs im Frühling und Herbst das nördliche Illinois.

XXXV. FAM. PHALAROPODIDAE.

Genus Steganopus Vieill.

179. S. Wilsonii Coues. Wassertreter, Wilson's/Wassertreter; Wilson's Phalarope. Brutvogel, jedoch selten beobachtet.

XXXVI. FAM. RECURVIROSTRIDAE.

Genus Recurvirostra Linné.

180. R. america na Gmel. Säbelschnäbler; American Avocet. Anscheinend selten und nur im Herbst beobachtet.

XXXVII. FAM. RALLIDAE.

a. Genus Rallus Bechstein.

181. R. elegans Aud. Wasserhuhn; Red-breasted Rail; King Rail, Mud Hen, Marsh Hen. In ausgedehnten wasserreichen, Sümpfen Brutvogel.

182. R. virginianus Linn. Virginische Ralle; Virginia Rail. Brutvogel, aber seltener als der vorige. Bewohnt dieselben Oertlichkeiten.

b. Genus Porzana Vieillot.

183. P. carolina Brd. Gemeines Sumpfhühnchen; Carolina Rail. Ziemlich zahlreicher Brutvogel in sumpfigen Gegenden, in mit Schilf, Wassergräsern und niederem Gestrüpp bestandenen Teichrändern.

184. P. noveboracensis Brd. Kleines gelbes Teichhühnchen; Little Yellow Rail. Brutvogel, aber seltener als der vorige.

185. P. jamaicensis Brd. Kleines schwarzes Teichhühnchen; Little Black Rail. Ziemlich zahlreicher Brutvogel, namentlich an kleinen mit Wasserlilien bewachsenen Teichen und Sümpfen.

c. Genus Gallinula Brisson.

186. G. galeata Licht. Moorhühnchen; Florida Gallinule. Ziemlich seltener Brutvogel.

\section{d. Genus Fulica Linné.}

187. F. americana Gmel. Amerikanisches Wasserhuhn; American Coot. Ziemlich zahlreicher Brutvogel. 


\section{FAM. GRUIDAE.}

Genus Grus Linné.

188. G. americana Temm. und

189. G. canadensis Temm. Nur während der Zugzeit beobachtet.

XXXIX. FAM. ANATIDAE.

a. Genus Chen Boie.

190. C. hyperboreus Boie. Schneegans; Snow Goose. Häufig während der Zugzeit.

191. C. c a erulescens Ridgw. Blaugans; Blue Goose. Zugzeit.

b. Genus Anser Linné.

192. A. albifrons Gambeli Coues. Blässengans; Whitefronted Goose. Kommt in der Zugzeit vor.

c. Genus Bernicla Stephens.

193. B. canadensis Boie. Schwanen- oder Canadagans; Canada Goose. Diese schöne Gans ist während der Zugzeit sehr zahlreich, doch dürfte sie jetzt nur noch in einzelnen abgelegenen Oertlichkeiten brüten.

d. Genus Anas Linné.

194. A. boschas Linn. Stockente; Mallard. Brutvogel.

195. A. obscura Gmel. Schwarze Stockente; Dusky Duck, Black Mallard. Seltener Brutvogel.

e. Chaulelasmus Gray.

196. C. streperus Gray. Mittel-, Schnatter- oder Lärmente; Gadwell. Während der Zugzeit nicht selten.

d. Genus Mareca Steph.

197. M. americana Selby. Amerikanische Pfeifente; American Widgeon, Baldpate. Häufiger Zugvogel, doch brütet sie hie und da an entlegenen, wasserreichen Oertlichkeiten.

$$
\text { e. Dafila Leach. }
$$

198. D. acuta Bonap. Spiessente; Pintail Duck. Nicht zahlreich; brütet in sumpfigen Gegenden, wo sich grosse Wasserflächen in der Nähe befinden.

\section{f. Genus Spatula Boie.}

199. S. clypeata Boie. Löffelente, Spatel- oder Schellente.

In seichten wasserreichen mit Schilf und allerlei Wasserpflanzen bestandenen Teichen und Sümpfen vorkommender Brutvogel. 
g. Genus Querquedula Stephens.

200. Q. discors Steph. Blauflügelige Krickente; Blue-winged Teal. Brutvogel, aber nur in der Zugzeit häufig.

h. Genus Nettion Kaup.

201. N. carolinensis Brd. Grünflügelige Krickente, Krautente. Zahlreich während der Zugzeit; kommt vielleicht auch an manchen Orten noch als Brutvogel vor.

i. Genus Fulix Sundevall. Fuligula Steph.

202. F. marila Brd. Berg-, Muschelente; Scaup Duck. Zugzeit. Selten.

203. F. affinis Brd. Kleine Moorente; Lesser Scaup Duck und

204. F. collaris Brd. Ringelmoorente; Ring-necked Duck. Nur auf den Märkten Chicagos gesehen.

k. Genus $A$ ix Boie.

205. A. sponsa Boie. Wald-, Braut- oder Sommerente; Wood Duck; Summer Duck. Diese schönste aller unserer Enten kommt nur in Waldgegenden in der Nähe von ausgedehnten wasserreichen Sümpfen und Teichen vor. Früher zahlreich, wird sie jetzt von Jahr zu Jahr seltener. Sie brütet in Baumhöhlungen des niedrigen, ausgedehnten Waldes.

\section{Genus Aethyia Boie.}

206. A. vallisneria Boie. Kanevasente; Canvass-back Duck. Diese schöne, grosse Ente, welche ihren wissenschaftlichen Namen von ihrer Hauptnahrung Vallisneria spiralis L., einer in langsam fliessenden und stagnanten Gewässern häufigen Pflanze, erhalten hat, ist während der Zugzeit ein ziemlich zahlreicher Vogel.

207. A. americana Bonap. Rothkopf; Red-head, American Pochard. Selten; nur in der Zugzeit.

m. Genus Clangula Flemming.

208. C. albeola Steph. Schellente, Büffelkopf; Buffle-head, Butter-ball, Di-Dipper. Spätherbst und frühzeitig im Frühling vorkommend.

n. Genus Histrionicus Lesson.

209. H. minutus Dresser. Harlekin-, Kragen- oder Stromente; Harlequin Duck. Im Winter vorkommend.

0. Genus Harelda Leach.

210. H. glacialis Leach. Eisente, Langschwanz; Longtailed Duck. Im Winter auf offenen Wasser. 
p. Genus So materia Leach.

211. S. mollissima Dresseri Coues. Amerikanische Eiderente; American Eider. Wintergast auf Lake Michigan.

q. Oidemia Flemming.

212. O. americana Sw. Trauer- oder Rabenente; BlackScoter. Im Winter auf dem Michigan.

r. Genus Melanetta Boie.

213. M. velvetina Brd. Sammetente; Velvet Scoter. Zahlreich auf dem Lake Michigan im Winter.

s. Genus Pelionetta Kaup.

214. P. perspicillata Kaup. Brillenente; Surf Duck, Sea Coot. Wintergast auf dem Michigansee.

t. Genus Erismatura Bonaparte.

215. E. rubida Bonap. Ruderente; Ruddy Duck. Eine der zahlreichsten Enten während der Brutzeit. Bevorzugt zum Aufenthalt wasserreiche, mit Binsen, Seggen, Schilf und Wasserlilien dicht bewachsene Prairiesümpfe und Teiche.

u. Genus Mergus Linné.

216. M. serrator Linn. Rothbrüstiger Säger, Sägetaucher; Redbreasted Merganser. Seltener Brutvogel, zahlreich während der Zugzeit.

\section{v. Genus Lophodytes Reichenbach.}

217. L. cucullatus Reich. Haubensäger; Stroded Merganser, Sheldrake. Brutvogel in sumpfigen Waldgegenden, aber nicht zahlreich.

XL. FAM. $L A R I D A E$.

a. Genus Riss a Leach.

218. R. tridactýla Bonap. Dreizehen-, Winter- oder Fischermöve; Kittiwoke Gull. Kommt als Strichvogel an die Küste des Michigan.

\section{b. Genus Larus Linné.}

219. L. glaucus Brünnich. Eismöve, Tauchermöve; Glaucous Gull. Wintergast; ebenso:

220. L. marinus L. Mantelmöve; Great Black-backed Gull und

221. L. leucopterus Faber. Polarmöve; White-headed Gull.

222. L. argentatus smithsonianus Coues. Amerikanische Heringsmöve; American Herring Gull. Häufiger Wintergast. e. Genus Xema Leach.

223. $X$. Sabinei Leach. Gabelschwanzmöve; Fork-tailed Gull. Sehr seltener Wintergast. 


\section{d. Genus Sterna Linné.}

224. S. anglica Mont. Lachseeschwalbe, Sumpfseeschwalbe; Marsh Tern. Sommergast, ebenso:

225. S. caspia Pall. Raubseeschwalbe; Caspian Tern und

226. S. regia Gamb. Königsseeschwalbe; Royal Tern.

227. S. fluviatilis Naum. Gemeine Seeschwalbe, Common Tern. Im Sommer zeitweise sehr häufig.

e. Genus Hydrochelidon Boie.

228. H. lariformis surinamensis Ridgw. Schwarze Seeschwalbe; Black Tern. Im Sommer am Michigansee ein sehr häufiger Vogel.

XLI. FAM. STERCORARIIDAE.

Genus Stercorarius Briss.

229. S. pomatorhinus Temm. Raubmöve, Skuamöve; Skua, Pomarine Jaëger. Wintergast am Michigansee; nicht zahlreich.

XLII. FAM. PODICIPEDIDAE.

a. Genus Dytes Kaup.

230. D. auritus Ridgw. Gehörnter Steissfuss, Horntaucher; Storned Grebe. Ziemlich zahlreich während der Zugzeit, sehr vereinzelt im Sommer.

\section{b. Genus Podylymbus Lesson.}

231. P. podiceps Lawr. Steissfuss, Taucher, Tauchente; Thick-billed Grebe, Water Witch, Dab Chick. In ausgedehnten sumpfigen Gegenden, wo eine grosse Anzahl verschiedener Wasserpflanzen beisammen stehen, Brutvogel. Bei Ansicht eines Menschen taucht er schneli unter und kommt gewöhnlich da wieder zum Vorschein, wo dichte Wasserpflanzen stehen.

XLIII. FAM. COLYMBIDAE.

Genus Colymbus Linné.

232. C. tor quatus Brünnich. Eistaucher, Gitterlumme; Great Northern Diver, Loon. Wintergast.

233. C. septentrionalis Linn. Rothhalsiger Taucher, rothhalsige Lumme, Nordseetaucher. Red-throated Diver. Wintergast.

Am Schlusse dieser „Beiträge zur Ornis des nördlichen Illinois“ möchte ich bemerken, dass die Zahl der Sumpf- und Wasservögel sicherlich grösser sein wird, als ich hier angegeben. Ich hatte nicht die Gelegenheit, diesen Vögeln mehr Aufmerksamkeit zu schenken. Auch möchte ich hier bemerken, dass gerade die ornithologische Literatur über Sumpf- und Wasservögel Nordamerikas grosse Lücken zeigt. Noch immer lässt der vierte Band der 
„History of North American Birds" (von Baird, Brewer und Ridgway) auf sich warten,*) obwohl seit Ausgabe des dritten Bandes acht Jahre verflossen sind. Das. Beste aus neuerer Zeit über diesen Zweig der Ornithologie findet sich in „Birds of the North-West" (1874) von unserem ausgezeichneten, unermïdlichen Dr. Elliott Coues und in C. J. Maynard's „Birds of Eastern Nord America“ (1879). Nachträgliche Bemerkungen.

Seite 412 des XXVIII. Bandes des „Journal für Ornithologie“ habe ich angegeben, dass Harporhynchus rufus und Galeoscoptes carolinensis gleichzeitig, etwa anfangs Mai, erscheinen. Hierauf schrieb mir Herr Otto Widmann von St. Louis (Mo.) Folgendes: „Es fiel mir auf, dass Sie sagen, „sie erscheint mit der Katzendrossel zu gleicher Zeit". Ich wusste nämlich, dass die Ankunftszeiten dieser beiden Arten weit auseinanderliegen, was St. Louis betrifft. Ich bin gewohnt, Harporhynchus rufus als einen der ersten Sänger zu begrüssen, wenn die Bäume noch kahl sind und Alles noch winterlich aussieht, während die Katzendrossel mit zu denen gehört, welche warten, bis die Sträucher grün sind. Im Jahre 1879 und 1880 sah ich den ersten Harp. rufus am 29. März, im Jahre 1878 sogar schon am 22. März. Freilich war das Frühjahr des Jahres 1878 ausserordentlich früh eingetreten, denn am 12. März war dieselbe Magnolie, die sonst Anfangs April, dieses Jahr (1881) erst am 24. April in Blüthe kam, schon in voller Blüthe. Unsere Katzendrossel kam jedoch, trotz allem schönen Wetter, auch im Jahre 1878 erst am 24. April, 1879 ebenfalls am 24. April. Im Jahre 1880 sah ich die erste schon am 18. und am 21. April hörte ich mehrere singen; dieses Jahr erschien sie erst am 25. April. - Ganz natürlich ist es, dass Chicago und St. Louis mit ihrem verschiedenen Klima auch verschiedene Ankunftszeiten aufweisen, und mag der Unterschied bei den frühankommenden ein weit grösserer sein, als bei den Ende April und Anfangs Mai Ankommenden. Die ersteren werden viel häufiger durch kaltes Wetter und Schnee zurïckgehalten, während die späteren meistens rasch die Reise nach dem Norden fortsetzen, also unter Umständen die anderen noch einholen können."

Wahrscheinlich kommt Harporhynchus rufus auch im nördlichen Illinois bedeutend früher an als Galeoscoptes carolinensis. Da es aber Anfangs April und oft auch noch Mitte April dort

*) Ist inzwischen in 2 Voll. unter dem Titel: The Water Birds of North America erschienen.

Der Herausgeber. 
recht winterliches Wetter ist, so sind diese Vögel in dem dichten Gestrüpp der Sümpfe und Flussniederungen jedenfalls meinen Beobachtungen entgangen.

Band XXIX (1881) p. 201 ist irrthümlich angegeben, dass Progne purpurea im nördlichen Illinois jährlich z w ei Bruten mache. Dieses geschieht jedoch nur, wenn die erste Brut verungliickte; nur e in e Brut findet in der Regel statt. Auch in St. Louis, etwa 300 englische Meilen südwestlich von Chicago, findet, nach den Angaben des Herrn Widmann, jährlich nur eine Brut statt.

\section{Nachtrag zur Ornis der Insel Salanga.}

Von

\section{Dr. August Müller in Frankfurt a/M.}

(Siehe Journal 1882, Seite 353 u. ff.)

Dem unermüdlichen Sammeleifer des Herrn Capt. Johannes Weber in Tongkah auf Salanga verdanken wir wieder reiche zoologische Sammlungen. Im Anschluss an meine frühere Arbeit*) lasse ich eine Aufzählung des in der letzten Sendung enthalten gewesenen ornithologischen Materials folgen. Dasselbe weist zehn für Salanga neue Arten**) auf. Auch die bereits von genannter Insel bekannten Species glaube ich von der Liste nicht ausschliessen zu dürfen und begründe dies einestheils in den vom Sammler sorgfältig notirten Daten, anderntheils aber auch in dem weiteren Messungsmateriale, was uns durch diese wiederholte Ausbeute geboten wurde.

Wer jemals zoogeographische Studien verfolgte, wird dies gerechtfertigt finden. Wie sehr Daten- und Messungs-Angaben aus Arbeiten über nächstliegende Gebiete späteren zoogeographischen Untersuchungen helfend und stützend an die Hand gehen, habe ich bei Bearbeitung des ersten Beitrages zur Ornis von Salanga zur Genüge erfahren. Für diesmal habe ich Angaben über geographische Verbreitungsverhältnisse unterlassen. Die Anzahl der für die Insel neủen Arten ist zu klein, und ich behalte mir dementsprechende Mittheilungen für später vor, wo ich hoffe, dass reicheres hierzu zu verwendendes Material vorliegt. Nur möchte ich nicht unterlassen, Herrn Capt. Weber für die aufopfernden Be-

*) Cab., Journ. f. Ornithol., p. 353-448.

**) In der systemat. Aufzählung mit vorgedrucktem * versehen. 


\section{$2 \mathrm{BHL}$ Biodiversity Heritage Library}

1885. "Beiträge zur Ornis des nördlichen Illinois." Journal fu

r Ornithologie 33, 142-151. https://doi.org/10.1007/bf02029097.

View This Item Online: https://www.biodiversitylibrary.org/item/101743

DOI: https://doi.org/10.1007/bf02029097

Permalink: https://www.biodiversitylibrary.org/partpdf/142332

\section{Holding Institution}

Smithsonian Libraries

\section{Sponsored by}

Biodiversity Heritage Library

\section{Copyright \& Reuse}

Copyright Status: Public domain. The BHL considers that this work is no longer under copyright protection.

This document was created from content at the Biodiversity Heritage Library, the world's largest open access digital library for biodiversity literature and archives. Visit BHL at https://www.biodiversitylibrary.org. 